\title{
Intraarterial administration of norcantharidin attenuates ischemic stroke damage in rodents when given at the time of reperfusion: novel uses of endovascular capabilities
}

\author{
Imad S. Khan, MD, ${ }^{1,2}$ Mitchell Odom, BS, ${ }^{3}$ Moneeb Ehtesham, MD, ${ }^{3}$ Daniel Colvin, $\mathrm{PhD},{ }^{4}$ \\ C. Chad Quarles, PhD, ${ }^{4}$ BethAnn McLaughlin, PhD, ${ }^{5}$ and Robert J. Singer, MD ${ }^{1,2}$

\begin{abstract}
1J.B. Marshall Laboratory for Neurovascular Therapeutics at Dartmouth; ${ }^{2}$ Division of Neurosurgery, Dartmouth-Hitchcock Medical Center, Lebanon, New Hampshire; ${ }^{3}$ Department of Neurosurgery, Vanderbilt University Medical Center; ${ }^{4}$ Vanderbilt University Institute of Imaging Sciences; and ${ }^{5}$ Department of Neurology and Pharmacology, Vanderbilt University School of Medicine, Nashville, Tennessee
\end{abstract}

\begin{abstract}
OBJECTIVE Matrix metalloprotease-9 (MMP-9) plays a critical role in infarct progression, blood-brain barrier (BBB) disruption, and vasogenic edema. While systemic administration of MMP-9 inhibitors has shown neuroprotective promise in ischemic stroke, there has been little effort to incorporate these drugs into endovascular modalities. By modifying the rodent middle cerebral artery occlusion (MCAO) model to allow local intraarterial delivery of drugs, one has the ability to mimic endovascular delivery of therapeutics. Using this model, the authors sought to maximize the protective potential of MMP-9 inhibition by intraarterial administration of an MMP-9 inhibitor, norcantharidin (NCTD).

METHODS Spontaneously hypertensive rats were subjected to 90 -minute MCAO followed immediately by local intraarterial administration of NCTD. The rats' neurobehavioral performances were scored according to the ladder rung walking test results and the Garcia neurological test for as long as 7 days after stroke. MRI was also conducted 24 hours after the stroke to assess infarct volume and BBB disruption. At the end of the experimental protocol, rat brains were used for active MMP-9 immunohistochemical analysis to assess the degree of MMP-9 inhibition.

RESULTS NCTD-treated rats showed significantly better neurobehavioral scores for all days tested. MR images also depicted significantly decreased infarct volumes and BBB disruption 24 hours after stroke. Inhibition of MMP-9 expression in the ischemic region was depicted on immunohistochemical analysis, wherein treated rats showed decreased active MMP-9 staining compared with controls.
\end{abstract}

CONCLUSIONS Intraarterial NCTD significantly improved outcome when administered at the time of reperfusion in a spontaneously hypertensive rat stroke model. This study suggests that supplementing endovascular revascularization with local neuroprotective drug therapy may be a viable therapeutic strategy.

http://thejns.org/doi/abs/10.3171/2015.4.JNS142400

KEY WORDS intraarterial therapy; MMP-9; stroke; ischemia reperfusion injury; norcantharidin; neuroprotection; vascular disorders

$\mathrm{F}$ OCAL cerebral ischemia and reperfusion has long been recognized to cause disruption of the bloodbrain barrier (BBB), which leads to the development of vasogenic edema and fosters hemorrhagic transformation. Accumulating evidence points toward the role of matrix metalloproteinases as one of the main mediators causing disruption. ${ }^{33}$ Specifically, matrix metalloprotease-9 (MMP-9) is known to break down a number of critical components of the BBB's basal lamina, including fibronectin, elastin, vitronectin, and Type IV collagen. ${ }^{19}$

The use of tissue plasminogen activator (tPA) has also been associated with an increased expression of MMP-9 as early as within 8 hours after onset of stroke. ${ }^{30}$ A relationship between the expression of MMP-9, the rate of hemorrhagic transformation, and clinical outcomes has been well established. ${ }^{2,16,25,26,34}$ Furthermore, increased levels of

ABBREVIATIONS BBB = blood-brain barrier; DMSO = dimethyl sulfoxide; ICA = internal carotid artery; IV = intravenous; $M C A=$ middle cerebral artery; $M C A O=M C A$ occlusion; MMP-9 = matrix metalloprotease-9; NCTD = norcantharidin; tPA = tissue plasminogen activator; VEGF = vascular endothelial growth factor. SUBMITTED October 20, 2014. ACCEPTED April 23, 2015.

INCLUDE WHEN CITING Published online November 6, 2015; DOI: 10.3171/2015.4.JNS142400. 
MMP-9 expression have been shown to be associated with the size of the lesion and an increased risk of death in patients..$^{14,16}$ The association between ischemic stroke, BBB disruption, and patient mortality makes MMP-9 a viable therapeutic target.

Among MMP-9 chemical inhibitors, norcatharidin (NCTD) has shown particular therapeutic promise. NCTD is a small-molecule, synthetic, demethylated analog of the naturally occurring cantharidin isolated from blister beetles. ${ }^{4}$ In both colorectal cancer and hepatocellular carcinoma cells, NCTD has shown specific MMP-9 activity via interaction and inhibition of its expression. ${ }^{3,41}$ Studies have demonstrated potent antimetastatic and antiangiogenic properties of NCTD in addition to its potential to generate tolerogenic skin grafts. ${ }^{15,17,20,41,42}$ It is eliminated in 6 hours from all tissues of rats and mice except the liver and kidneys. ${ }^{8}$ The dose used in this work has been shown to block both MMP-9 as well as hypervascularization and the associated MAPK/vascular endothelial growth factor (VEGF) pathways in vitro and in vivo. ${ }^{43}$ More importantly, NCTD crosses the BBB, making it a suitable candidate for neurotherapeautics. ${ }^{5}$

Among the various management strategies for acute ischemic stroke, endovascular modalities have seen considerable interest. ${ }^{24}$ Endovascular thrombectomy allows expeditious reperfusion and can also theoretically allow selective infusion of potential neuroprotective agents to the ischemic cerebral region. There is, however, a paucity of research regarding the use of drugs that may be administered locally (intraarterially) at the time of reperfusion to provide neuroprotection. By the modification of the rodent middle cerebral artery (MCA) occlusion (MCAO) model to allow intraarterial drug delivery, we have the ability to mimic endovascular delivery of drugs in a patient with ischemic stroke. In this study we investigated the neuroprotective efficacy of intraarterial NCTD in ischemia and subsequent reperfusion.

\section{Methods}

\section{Animals}

Adult male spontaneously hypertensive rats between 12 and 14 weeks of age and weighing 290-310 g (obtained from Charles River) were used for this study. Animals were housed under standard conditions $\left(21^{\circ}-23^{\circ} \mathrm{C}, 12-\right.$ hour light-dark cycle) with unlimited access to standard food and water. All animal experiments were approved by the Vanderbilt University Institutional Animal Care and Use Committee and performed according to the National Research Council's Guide for the Care and Use of Laboratory Animals.

\section{Animal Preparation and Surgery}

A total of 40 rats were weighed and randomly assigned to 1 of 3 groups: treated $(90$-minute MCAO followed by intraarterial drug administration), controls (MCAO followed by intraarterial vehicle administration), and shams (surgery but no MCAO). The MCAO surgery was conducted as previously described with a few modifications. ${ }^{21}$ Briefly, the right-sided common, internal, and external carotid arteries were exposed and their tributaries cau- terized. An intraluminal suture (Doccol Corp.) was then routed into the internal carotid artery (ICA) via the external carotid artery and pushed to occlude the MCA.

Subsequently, anesthesia was removed and only rats showing left-sided weakness on the upright test were included in this study. Surgery in the sham surgery group was similar to the one described above, with the key difference that intraluminal suture was advanced to occlude the MCA for less than 1 minute before being removed.

After a stroke time of 90 minutes the rats were reanesthetized and the suture was removed. The external carotid artery stump used for the intraluminal suture was then used to introduce a polyethylene-20 tube that was then routed into the proximal ICA; this was subsequently used for intraarterial drug administration. Additionally, 10 rats (5 treated and 5 controls) had external jugular catheters inserted for MRI contrast studies.

\section{Drug Administration}

Rats were randomized into treated and control groups. NCTD $(0.5 \mathrm{mg} / \mathrm{kg})$ dissolved in dimethyl sulfoxide (DMSO) and diluted in normal saline (in a 1 to 5 ratio of DMSO to saline) was then infused directly into the ipsilateral ICA using an infusion pump (Harvard Apparatus) at a rate of $0.10 \mathrm{ml} / \mathrm{min}$. The dosage of NCTD was adapted from a study that showed a positive biological response with the same dose when administered intraarterially for rat hepatoma. ${ }^{22}$ The control rats were administered normal saline and DMSO (1:5) without any drug at the same infusion rate. Diluted DMSO was administered to both control and treated rats to control for any effects DMSO might have on the BBB ${ }^{18}$ Infusion lasted from 5 to 7 minutes.

\section{Sensorimotor Function Assessment}

For the ladder rung walking test, the rats were trained 2 times prior to stroke induction. Training sessions were spaced 24 hours apart and the second practice session was conducted at least 24 hours prior to the induction of stroke. The timeframe was consistent between all rats and all rats had consistent practice sessions. The rats were walked through the ladder rung 3 times after stroke for neurological assessment (poststroke Days 1, 3, and 7) in which average foot placement accuracy and foot fault scores were measured (Videos 1 and 2).

VIDEO 1. Clip showing a nontreated (control) rat undergoing the ladder rung walking test on poststroke Days 1,3, and 7. Copyright Imad S. Khan. Published with permission. Click here to view.

VIDEO 2. Clip showing a treated rat undergoing the ladder rung walking test on poststroke Days 1, 3, and 7. Copyright Imad S. Khan. Published with permission. Click here to view.

The animals were also scored daily according to the Garcia Neurological Test for as many as 7 days after stroke. ${ }^{11}$ All the behavioral scoring was performed in a double-blinded manner. The experimental design is shown in Fig. 1A. Details are available in the Supplemental Information.

\section{Imaging}

MRI was performed 24 hours following MCAO. Infarct volumes and BBB integrity were measured using 
A

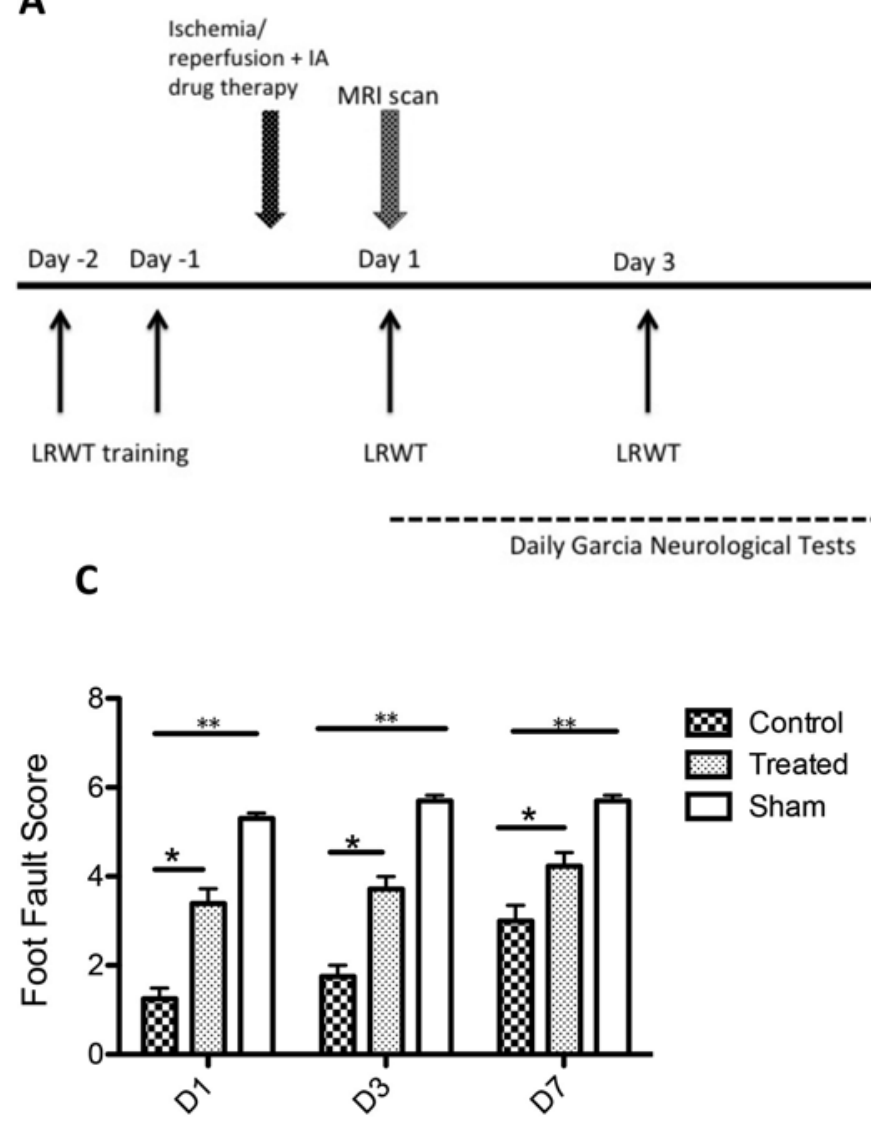

B

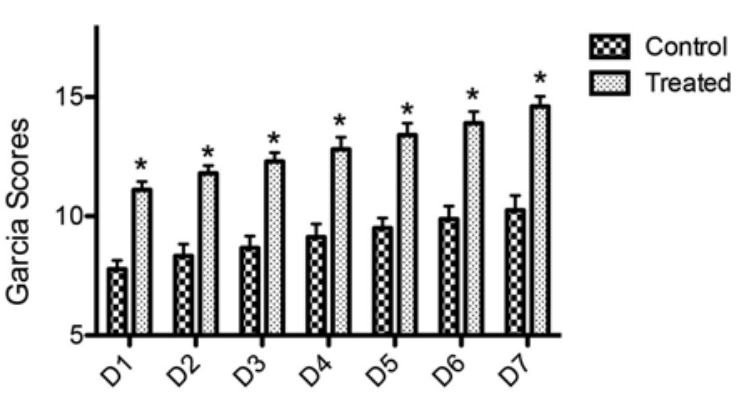

FIG. 1. A: Experimental design of the study. IA = intraarterial; LRWT = ladder rung walking test. B: NCTD treated rats had significantly higher Garcia scores compared with vehicle-injected controls on all days assessed ( ${ }^{*} p<0.0001, n=11 /$ group). C and D: Following ischemic stroke, NCTD-treated rats had significantly higher average foot fault scores (C) and a significantly lower proportion of missteps (D) compared with vehicle-injected rats at all days tested ( ${ }^{*} p<0.05, n=11 /$ group). Both the 90-minute MCAO groups performed worse than animals receiving sham surgery in markers of foot fault scores and proportion of missteps $\left({ }^{* *} p<0.001\right.$, sham $\left.n=5\right)$.

diffusion weighted and dynamic contrast enhanced imaging, respectively. Blinded observers performed the image analyses. Details are available in the Supplemental Information.

\section{Immunohistochemical Analysis}

To qualitatively assess the expression of active MMP-9 after treatment in animals, immunohistochemical analysis was performed in 6 rats ( 3 treated and control each). Brains from rats on stroke postoperative Day 7 were sectioned and were incubated with primary anti-active MMP-9 antibody (1:200 dilution, Novus Biologicals). Details are available in the Supplemental Information.

\section{Statistical Analysis and Sample Size Estimation}

Data represent the mean \pm standard error of the mean (SEM). The Student t-test was used for comparison between 2 groups, and an ANOVA for multiple comparisons with a post hoc Tukey test was used after validating the normal distribution of these data sets using the Kolmogorov-Smirnov test. Spearman's rank correlation coefficient was used to test the association between infarct volume and ladder rung walking test scores.
For power analysis, we estimated a $30 \%$ improvement in sensorimotor score (primary outcome) and a $15 \%$ decrease in infarct volume and BBB permeability with treatment (secondary outcome), based on our pilot study results. Using a standard deviation of $20 \%$ of the mean within each group, $\alpha=0.05$, the sample size to achieve power $(1-\beta)=$ 0.8 was at least 10 per group.

\section{Results}

Prior to the MCAO surgery, none of the rats had any prior interventions and showed no neurological dysfunction on testing. There were a total of 2 premature deaths: 1 each in the treated and control groups (death on poststroke Day 1 in both instances). Both deaths were due to hemorrhagic transformation of the ischemic stroke (observed on gross examination of the brain). Of a total of 15 rats in the treated and control groups, complete behavioral data were available for 14 animals (93.3\%). While we do not perform long-term toxicity and behavioral studies, we observed no specific adverse effects as a result of NCTD administration and the experimental protocols were not altered during the course of the study 


\section{Effect of NCTD on Sensorimotor Functions}

All animals with 90-minute MCAO showed a significant sensorimotor deficit (scored 3 points on the Benderson Scale) prior to reperfusion, demonstrating successful MCAO. To assess the neurological outcome in the rats we evaluated them using the Garcia neurological test and tested fine and gross motor functions via the ladder rung walking test. The Garcia test was administered daily for 7 days by blinded observers. For all days tested, the treated rats scored significantly higher compared with controls (Fig. 1B). None of the treated or untreated rats deteriorated after the stroke, and showed improving scores consistent with recovery.

The ladder rung walking test, used to assess forelimb fault and placement accuracy scores, was administered on poststroke Days 1, 3, and 7 (Fig. 1C and D). The treated rats displayed significantly higher average forelimb fault scores on all days tested, signifying better placement accuracy. Moreover, treated rats displayed significantly better stability compared with control rats (fewer missteps) on all days tested. Rats undergoing sham surgery did not display any neurological deficits and had the best accuracy and fewest missteps compared with both the treated and control groups ( $\mathrm{p}<0.001$ for all days tested).

\section{Effect of NCTD on Infarct Volume and BBB Disruption}

To compare brain infarct volumes between the treated and control groups we calculated infarct volumes using diffusion weighted imaging scans conducted 24 hours after the onset of stroke. The infarct volumes were calculated as the sum of the number of voxels within the segmented infarct region multiplied by the volume of each voxel. Treated rats had significantly reduced average infarction volume compared with control rats $\left(148.1 \pm 10.2 \mathrm{~mm}^{3} \mathrm{vs}\right.$ $178.5 \pm 9.9 \mathrm{~mm}^{3}, \mathrm{p}<0.05, \mathrm{n}=10$ per group; Fig. $\left.2 \mathrm{~A}\right)$, indicating a neuroprotective effect of NCTD after reperfusion. Treated rats, compared with controls, depicted variable cortical sparing which accounted for the decreased infarct volumes observed in this group.

To assess whether our treatment with NCTD affected
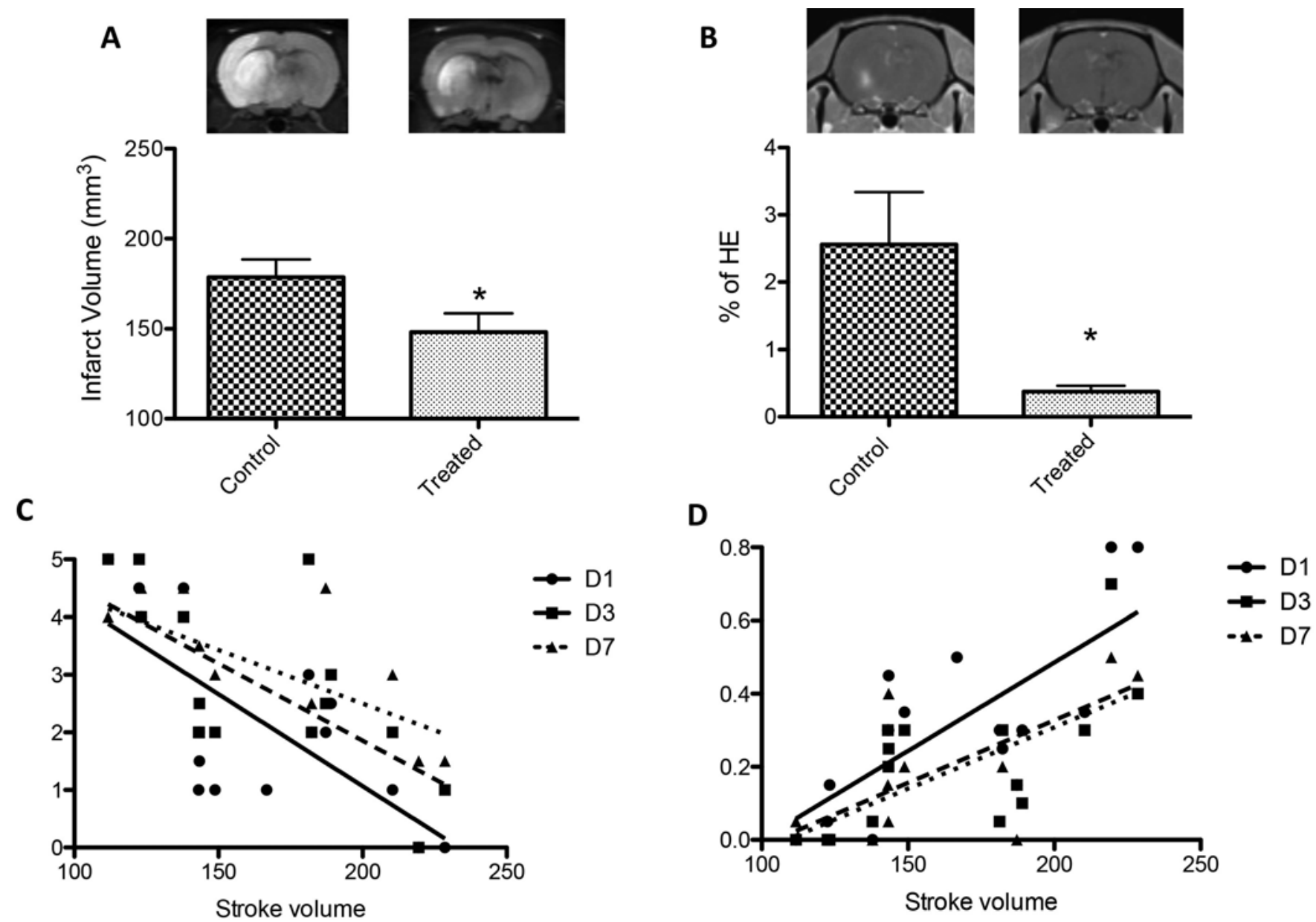

FIG. 2. A: NCTD-treated rats had decreased infarct volumes compared with vehicle-treated rats also exposed to 90 minutes of MCAO ( $\left.{ }^{*} p<0.05, n=10 / g r o u p\right)$. B: NCTD treatment was associated with a decreased percentage of regional contrast enhancement compared with the vehicle-treated MCAO group ( ${ }^{*} p<0.05, n=5 /$ group). $\mathrm{HE}=$ hemispheric enhancement. C: A significant negative correlation was found for stroke volumes versus forelimb fault scores for all days tested (Day 1: Spearman's $r=-0.6920$, $p=0.0030$; Day 3: Spearman's $r=-0.6748, p=0.0058$; Day 7: Spearman's $r=-0.6534, p=0.011$ ). $\mathbf{D}$ : A significant positive correlation was found for stroke volumes versus placement accuracy scores (Day 1: Spearman's $r=0.6850, p=0.0034$; Day 3: Spearman's $r=0.7430, p=0.0016$; Day 7: Spearman's $r=0.7470, p=0.0021$ ). 
the degree of BBB disruption after an episode of ischemia and reperfusion, we conducted dynamic contrast-enhanced scans 24 hours after the onset of stroke. Subsequently, we calculated the area of leakage (contrast enhancement) in the region of the infarct from enhancing pixels on permeability maps. The region of leakage was then expressed as a percentage of the area of ipsilateral hemisphere (designated as the percentage of hemispheric contrast enhancement). No contrast enhancement was observed in the nonischemic hemispheres in any of the rats. The percentage of hemispheric contrast enhancement was significantly reduced in treated rats compared with the control rats $(0.3 \%$ $\pm 0.01 \%$ vs $2.5 \% \pm 0.80 \%$, respectively; $\mathrm{p}<0.05, \mathrm{n}=5$ per group; Fig. 2B), indicating the inhibitory effect of NCTD on BBB permeability.

To evaluate how closely the stroke volume was related to ladder rung walking test results (forelimb fault and placement accuracy scores), we grouped the stroke volumes of all rats and plotted them against their respective forelimb fault and placement accuracy scores (Fig. 2C and D). A significant negative correlation was found for stroke volumes versus forelimb fault scores for all days tested ( $\mathrm{p}$ $<0.05$; Fig. 2C). Additionally, a significant positive correlation was found for stroke volumes versus placement accuracy scores ( $\mathrm{p}<0.05$; Fig. 2D).

\section{Immunohistochemical Analysis}

To assess the expression of MMP-9, immunohistochemical analysis was conducted using rat brains harvested at the end of the experimental protocol (poststroke Day 7; Fig. 3). The control group showed strong active MMP-9 staining throughout the ischemic cortex. This was especially evident in the endothelial cells. In contrast, the rat brains harvested from treated rats showed significantly decreased expression of active MMP-9, including in the endothelial cells. These results depicted the strong inhibitory action of NCTD on the expression of active MMP-9 after ischemic stroke.

\section{Discussion}

The use of intravenous (IV) tPA has become the standard of care for ischemic stroke after the landmark trial of the National Institutes of Neurological Disorders and Stroke demonstrated an improvement in the 3-month outcome if it was administered within 3 hours of the onset of symptoms.$^{28}$ Further research on the thrombolytic efficacy of IV tPA showed that while this treatment has high revascularization rates for distal thrombi, the revascularization rates of proximal thrombi (the ICA terminus, proximal MCA, vertebral arteries, and basilar artery) were much inferior. As higher revascularization rates are associated with better outcomes, the efficacy of IV tPA is significantly lower in patients who present with ICA or proximal MCA occlusions. ${ }^{36,38}$ This has led to an increased interest in the development of endovascular techniques to recanalize large vessel occlusions. ${ }^{24}$

Compared with IV thrombolysis, contemporary endovascular techniques show higher rates of revascularization for large vessel occlusions. ${ }^{32,37}$ Additionally, these modalities can be used to gain direct access to the region of stroke to deliver focused neuroprotective therapeutics. In this setting, the supplementation of endovascular thrombolysis with a neuroprotective agent may be able to shield the brain from the temporary but destructive effects of reperfusion.

MMP-9 has been recognized as a key target in the development of neuroprotective agents for acute ischemic stroke.,27 Expression of MMP-9 increases after cerebral ischemia and plays an important role in the direct disruption of the BBB by breaking down collagen Type 4, an integral part of the laminin forming the BBB and increasing the risk of hemorrhagic transformation..$^{12,33,35}$ MMP-9 knockout mice show significantly smaller infarct volumes and decreased BBB disruption than wild-type mice after stroke. ${ }^{1}$ Based on these findings we chose MMP-9 as a viable therapeutic target for intraarterial therapy. Our data suggest that intraarterial NCTD decreased the expression
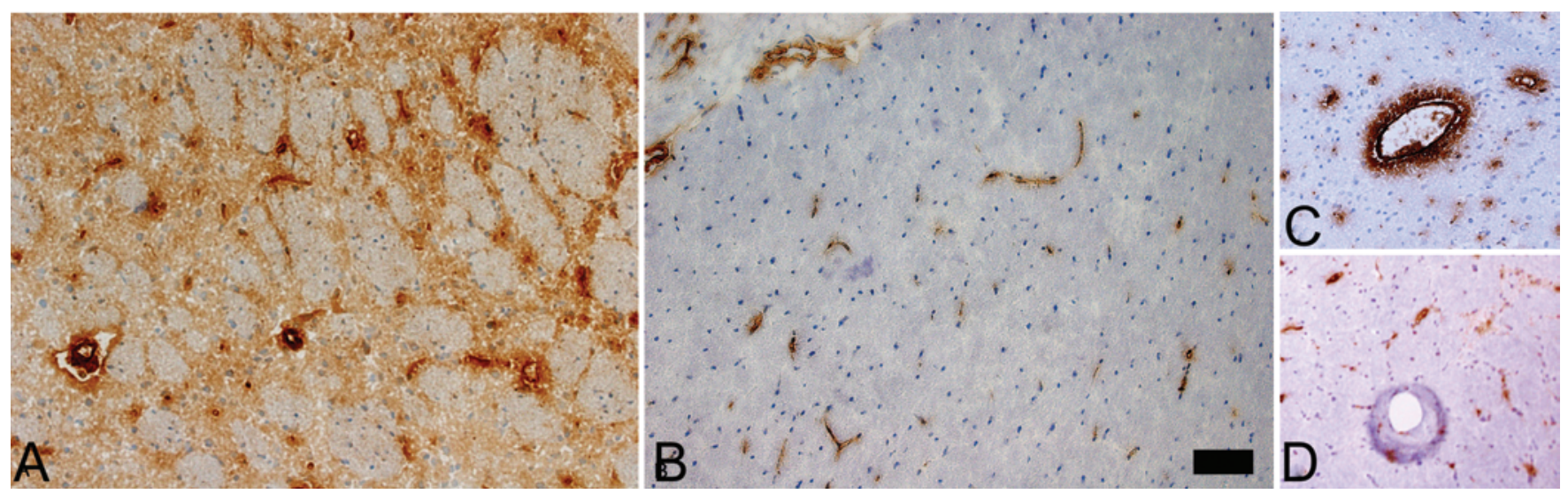

FIG. 3. Representative sections showing immunohistochemical staining of the ischemic rat brain 7 days after MCAO. Active MMP9 expression was not observed in contralateral control brain but was identified within and at the periphery of the ischemic cortical region. A: Extensive MMP-9 staining was evident in the cortical region of the untreated group. B: MMP-9 staining was less evident in the cortical region of the treated group. C: Strong MMP-9 endothelial staining was observed in the ischemic cortex of the untreated group. D: Endothelial staining was significantly attenuated in the cortex of the treated cohort. No staining was identified in ipsilateral or contralateral cortices of sham rats $(n=3$; data not shown). Original magnification $\times 20$. Bar $=250 \mu \mathrm{m}$. Figure is available in color online only. 
of MMP-9, provided robust cell preservation, increased BBB integrity, and improved sensorimotor outcomes. Furthermore, we did not recognize any adverse effects from our therapy, either due to the route of our delivery or the mechanism of action of the drug.

While it is important to recognize the destructive role that MMP-9 plays in the pathogenesis of ischemic stroke, emerging evidence suggests that the enzyme may have a more nuanced role. Data from Zhao and colleagues revealed temporal and spatial differences in the expression of MMP-9 after ischemic stroke: early MMP-9 signal localized in the brain endothelium, but by 7 days the MMP9 signal shifted to the periphery of cortical infraction At this time MMP-9 activates VEGF, leading to brain regeneration. ${ }^{44}$ Crucially, they reported that while a synthetic MMP-9 inhibitor could decrease infarct volumes if administered within 24 hours after stroke, delayed administration of MMP-9 inhibitors (7 days after stroke) disturbed the recovery of the brain, leading to worse outcomes and increased risk of hemorrhage in the ischemic cavity. ${ }^{44}$ Similarly, when Sood and colleagues administered a broad spectrum MMP-9 inhibitor (B-1101) 48 hours after stroke in a rodent stroke model, they reported significant neurological long-term deterioration in the treated group..$^{39}$

These findings underscore the importance of acute short-term inhibition of MMP-9 so as to not hinder recovery. It now appears that the therapeutic window for MMP9 inhibition may be less than 24-48 hours after stroke. ${ }^{45}$ With these considerations, a single-dose intraarterial delivery of MMP-9 inhibitors at the time of reperfusion is an attractive treatment strategy. As none of our treated rats deteriorated after treatment and showed improving sensorimotor scores consistent with recovery, our results suggest that we were able to successfully inhibit the early MMP-9 overexpression without impeding the repair processes.

It is also important to point out some of the advantages of local (intraarterial) therapy. Marinov and colleagues used preischemia intraarterial delivery of magnesium sulfate to show robust neuroprotective efficacy in a reversible focal ischemia model. ${ }^{23}$ Additionally, selective brain cooling to provide neuroprotection in stroke can also be achieved by intraarterial cold infusions. ${ }^{9}$ Compared with the systemic route, the requirement of a lower drug dose with intraarterial therapy was demonstrated by Woitznik and Schilling, who found a greater effect of a consistent dose of MK-801 (a glutamate receptor antagonist) when it was administered locally compared with intravenously. ${ }^{40}$ The higher bioavailability with intraarterial delivery systems has also been reported with stem cell therapeutics. ${ }^{31}$ Chemotherapeutic agents with a rapid systemic clearance also benefit from intraarterial administration. The higher bioavailability and high intratumoral concentrations achieved through an endovascular route have made intraarterial chemotherapy a viable option for CNS tumors and retinoblastomas. ${ }^{13,29}$ Systemic side effects of the drug may also be limited because of low dosage requirements through this route. Therefore, intraarterial delivery of drugs may provide an appealing avenue to test potential neuroprotective agents in which investigators may be able to maximize efficacy while minimizing systemic side effects. ${ }^{6}$

Our experimental design also has some advantages.
MR images for the assessment of BBB disruption and infarct volume allowed us to use the same rats for neurobehavioral scores, which meant that fewer rats were required overall. Such a design also permitted the correlation of infarct volumes with behavioral scores. Additionally, the spontaneously hypertensive rat was chosen as a model in keeping with the criteria set forth by the Stroke Therapy Academic Industry Roundtable (STAIR) working group, which developed guidelines for reproducible and predictive models of stroke for therapeutic development. ${ }^{10}$

We recognize that a shortcoming of our study design is that we chose a timeline (reperfusion after 90 minutes with drug delivery) that may not be very feasible in clinical practice. Our study investigated the proof of concept evidence that intraarterial administration of this selective MMP-9 inhibitor is capable of blocking degeneration and improving behavioral outcomes when administered at the time of reperfusion. Further research in our lab will study the effect of the drug with longer stroke times.

\section{Conclusions}

We conducted a preclinical study assessing the efficacy of intraarterial NCTD administered at the time of reperfusion in a rodent model to simulate endovascular treatment in a patient who undergoes revascularization and local therapy. Our findings require further investigation but may point toward the potential efficacy of administering neuroprotective agents endovascularly at the time of reperfusion to shield patients from the neurotoxic consequences of ischemia and reperfusion.

\section{Acknowledgments}

This work was funded by a grant from the Suzanne and Walter Scott Foundation and grant no. 1UH2NS080701 from the NIH.

\section{References}

1. Asahi M, Asahi K, Jung JC, del Zoppo GJ, Fini ME, Lo EH: Role for matrix metalloproteinase 9 after focal cerebral ischemia: effects of gene knockout and enzyme inhibition with BB-94. J Cereb Blood Flow Metab 20:1681-1689, 2000

2. Castellanos M, Leira R, Serena J, Pumar JM, Lizasoain I, Castillo J, et al: Plasma metalloproteinase-9 concentration predicts hemorrhagic transformation in acute ischemic stroke. Stroke 34:40-46, 2003

3. Chen YJ, Chang WM, Liu YW, Lee CY, Jang YH, Kuo CD, et al: A small-molecule metastasis inhibitor, norcantharidin, downregulates matrix metalloproteinase- 9 expression by inhibiting Spl transcriptional activity in colorectal cancer cells. Chem Biol Interact 181:440-446, 2009

4. Chen YJ, Kuo CD, Chen SH, Chen WJ, Huang WC, Chao $\mathrm{KSC}$, et al: Small-molecule synthetic compound norcantharidin reverses multi-drug resistance by regulating Sonic hedgehog signaling in human breast cancer cells. PLoS One 7:e37006, 2012

5. Cimmino F, Scoppettuolo MN, Carotenuto M, De Antonellis P, Dato VD, De Vita G, et al: Norcantharidin impairs medulloblastoma growth by inhibition of $\mathrm{Wnt} / \beta$-catenin signaling. J Neurooncol 106:59-70, 2012

6. Dang S, Liu X, Fu P, Gong W, Yan F, Han P, et al: Neuroprotection by local intra-arterial infusion of erythropoietin after focal cerebral ischemia in rats. Neurol Res 33:520-528, 2011

7. Dejonckheere E, Vandenbroucke RE, Libert C: Matrix metalloproteinases as drug targets in ischemia/reperfusion injury. Drug Discov Today 16:762-778, 2011 
8. Ding XY, Hong CJ, Liu Y, Gu ZL, Xing KL, Zhu AJ, et al: Pharmacokinetics, tissue distribution, and metabolites of a polyvinylpyrrolidone-coated norcantharidin chitosan nanoparticle formulation in rats and mice, using LC-MS/MS. Int J Nanomedicine 7:1723-1735, 2012

9. Esposito E, Ebner M, Ziemann U, Poli S: In cold blood: intraarteral cold infusions for selective brain cooling in stroke. J Cereb Blood Flow Metab 34:743-752, 2014

10. Fisher M, Feuerstein G, Howells DW, Hurn PD, Kent TA, Savitz SI, et al: Update of the stroke therapy academic industry roundtable preclinical recommendations. Stroke 40:2244-2250, 2009

11. Garcia JH, Wagner S, Liu KF, Hu XJ: Neurological deficit and extent of neuronal necrosis attributable to middle cerebral artery occlusion in rats. Statistical validation. Stroke 26:627-635, 1995

12. Gasche Y, Fujimura M, Morita-Fujimura Y, Copin JC, Kawase M, Massengale J, et al: Early appearance of activated matrix metalloproteinase- 9 after focal cerebral ischemia in mice: a possible role in blood-brain barrier dysfunction. J Cereb Blood Flow Metab 19:1020-1028, 1999

13. Gobin YP, Dunkel IJ, Marr BP, Brodie SE, Abramson DH: Intra-arterial chemotherapy for the management of retinoblastoma: four-year experience. Arch Ophthalmol 129:732737, 2011

14. Graham CA, Chan RWY, Chan DYS, Chan CPY, Wong LKS, Rainer TH: Matrix metalloproteinase 9 mRNA: an early prognostic marker for patients with acute stroke. Clin Biochem 45:352-355, 2012

15. Hsieh CH, Liao HF, Kuo CD, Huang YC, Shueng PW, Hsu YP, et al: Norcantharidin modulates development of dendritic cells and prolongs skin allograft survival. Transplantation 92:848-857, 2011

16. Inzitari D, Giusti B, Nencini P, Gori AM, Nesi M, Palumbo $\mathrm{V}$, et al: MMP9 variation after thrombolysis is associated with hemorrhagic transformation of lesion and death. Stroke 44:2901-2903, 2013

17. Jiang YM, Meng ZZ, Yue GX, Chen JX: Norcantharidin induces HL-60 cells apoptosis in vitro. Evid Based Complement Alternat Med 2012:154271, 2012

18. Kleindienst A, Dunbar JG, Glisson R, Okuno K, Marmarou A: Effect of dimethyl sulfoxide on blood-brain barrier integrity following middle cerebral artery occlusion in the rat. Acta Neurochir Suppl 96:258-262, 2006

19. Labrie M, St-Pierre Y: Epigenetic regulation of mmp-9 gene expression. Cell Mol Life Sci 70:3109-3124, 2013

20. Liu D, Shi P, Yin X, Chen Z, Zhang X: Effect of norcantharidin on the human breast cancer Bcap-37 cells. Connect Tissue Res 53:508-512, 2012

21. Longa EZ, Weinstein PR, Carlson S, Cummins R: Reversible middle cerebral artery occlusion without craniectomy in rats. Stroke 20:84-91, 1989

22. Mack P, Ha XF, Cheng LY: Efficacy of intra-arterial norcantharidin in suppressing tumour 14C-labelled glucose oxidative metabolism in rat Morris hepatoma. HPB Surg 10:65-72, 1996

23. Marinov MB, Harbaugh KS, Hoopes PJ, Pikus HJ, Harbaugh RE: Neuroprotective effects of preischemia intraarterial magnesium sulfate in reversible focal cerebral ischemia. J Neurosurg 85:117-124, 1996

24. Mokin M, Khalessi AA, Mocco J, Lanzino G, Dumont TM, Hanel RA, et al: Endovascular treatment of acute ischemic stroke: the end or just the beginning? Neurosurg Focus 36(1):E5, 2014

25. Montaner J, Alvarez-Sabín J, Molina CA, Anglés A, Abilleira $\mathrm{S}$, Arenillas J, et al: Matrix metalloproteinase expression is related to hemorrhagic transformation after cardioembolic stroke. Stroke 32:2762-2767, 2001

26. Montaner J, Molina CA, Monasterio J, Abilleira S, Arenillas
JF, Ribó M, et al: Matrix metalloproteinase-9 pretreatment level predicts intracranial hemorrhagic complications after thrombolysis in human stroke. Circulation 107:598-603, 2003

27. Morancho A, Rosell A, García-Bonilla L, Montaner J: Metalloproteinase and stroke infarct size: role for anti-inflammatory treatment? Ann N Y Acad Sci 1207:123-133, 2010

28. National Institute of Neurological Disorders and Stroke rt-PA Stroke Study Group: Tissue plasminogen activator for acute ischemic stroke. N Engl J Med 333:1581-1588, 1995

29. Newton HB: Intra-arterial chemotherapy of primary brain tumors. Curr Treat Options Oncol 6:519-530, 2005

30. Ning M, Furie KL, Koroshetz WJ, Lee H, Barron M, Lederer M, et al: Association between tPA therapy and raised early matrix metalloproteinase- 9 in acute stroke. Neurology 66:1550-1555, 2006

31. Pendharkar AV, Chua JY, Andres RH, Wang N, Gaeta X, Wang $\mathrm{H}$, et al: Biodistribution of neural stem cells after intravascular therapy for hypoxic-ischemia. Stroke 41:20642070, 2010

32. Penumbra Pivotal Stroke Trial Investigators: The penumbra pivotal stroke trial: safety and effectiveness of a new generation of mechanical devices for clot removal in intracranial large vessel occlusive disease. Stroke 40:2761-2768, 2009

33. Rosell A, Cuadrado E, Ortega-Aznar A, Hernández-Guillamon M, Lo EH, Montaner J: MMP-9-positive neutrophil infiltration is associated to blood-brain barrier breakdown and basal lamina type IV collagen degradation during hemorrhagic transformation after human ischemic stroke. Stroke 39:1121-1126, 2008

34. Rosell A, Ortega-Aznar A, Alvarez-Sabín J, FernándezCadenas I, Ribó M, Molina CA, et al: Increased brain expression of matrix metalloproteinase- 9 after ischemic and hemorrhagic human stroke. Stroke 37:1399-1406, 2006

35. Rosenberg GA, Estrada EY, Dencoff JE: Matrix metalloproteinases and TIMPs are associated with blood-brain barrier opening after reperfusion in rat brain. Stroke 29:2189-2195, 1998

36. Saqqur M, Uchino K, Demchuk AM, Molina CA, Garami Z, Calleja S, et al: Site of arterial occlusion identified by transcranial Doppler predicts the response to intravenous thrombolysis for stroke. Stroke 38:948-954, 2007

37. Saver JL, Jahan R, Levy EI, Jovin TG, Baxter B, Nogueira $\mathrm{RG}$, et al: Solitaire flow restoration device versus the Merci Retriever in patients with acute ischaemic stroke (SWIFT): a randomised, parallel-group, non-inferiority trial. Lancet 380:1241-1249, 2012

38. Sillanpää N, Saarinen JT, Rusanen H, Elovaara I, Dastidar P, Soimakallio S: Location of the clot and outcome of perfusion defects in acute anterior circulation stroke treated with intravenous thrombolysis. AJNR Am J Neuroradiol 34:100-106, 2013

39. Sood RR, Taheri S, Candelario-Jalil E, Estrada EY, Rosenberg GA: Early beneficial effect of matrix metalloproteinase inhibition on blood-brain barrier permeability as measured by magnetic resonance imaging countered by impaired longterm recovery after stroke in rat brain. J Cereb Blood Flow Metab 28:431-438, 2008

40. Woitzik J, Schilling L: A new method for superselective middle cerebral artery infusion in the rat. J Neurosurg 106:872-878, 2007

41. Yeh CB, Hsieh MJ, Hsieh YH, Chien MH, Chiou HL, Yang SF: Antimetastatic effects of norcantharidin on hepatocellular carcinoma by transcriptional inhibition of MMP-9 through modulation of NF-kB activity. PLoS One 7:e31055, 2012

42. Zhang JT, Fan YZ, Chen CQ, Zhao ZM, Sun W: Norcantharidin: a potential antiangiogenic agent for gallbladder cancers in vitro and in vivo. Int J Oncol 40:1501-1514, 2012 
43. Zhang L, Ji Q, Liu X, Chen X, Chen Z, Qiu Y, et al: Norcantharidin inhibits tumor angiogenesis via blocking VEGFR2/ MEK/ERK signaling pathways. Cancer Sci 104:604-610, 2013

44. Zhao BQ, Wang S, Kim HY, Storrie H, Rosen BR, Mooney DJ, et al: Role of matrix metalloproteinases in delayed cortical responses after stroke. Nat Med 12:441-445, 2006

45. Zlokovic BV: Remodeling after stroke. Nat Med 12:390-391, 2006

\section{Disclosure}

The authors report no conflict of interest concerning the materials or methods used in this study or the findings specified in this paper.

\section{Author Contributions}

Conception and design: Singer, Khan, Ehtesham. Acquisition of data: Khan, Odom, Colvin, Quarles. Analysis and interpretation of data: Khan, Odom, Colvin, Quarles. Drafting the article: Khan, Odom. Critically revising the article: Singer, McLaughlin. Reviewed submitted version of manuscript: Singer, Khan, McLaughlin. Approved the final version of the manuscript on behalf of all authors: Singer. Statistical analysis: Khan. Administrative/technical/material support: Singer, Ehtesham, Colvin,
Quarles, McLaughlin. Study supervision: Singer, Ehtesham, Colvin, Quarles, McLaughlin.

\section{Supplemental Information \\ Videos}

Video 1. https://vimeo.com/142533376.

Video 2 . https://vimeo.com/142533374.

\section{Online-Only Content}

Supplemental material is available with the online version of the article.

Supplemental Information. http://thejns.org/doi/suppl/10.3171/ 2015.4.JNS142400.

\section{Previous Presentation}

A portion of this work was presented as an oral presentation at the AANS/CNS Cerebrovascular Section Annual Meeting and the 4th SNIS International Endovascular Stroke Conference (IESC), in San Diego, California, February 10-11, 2014.

\section{Correspondence}

Robert J. Singer, Section of Neurosurgery, Geisel School of Medicine at Dartmouth, 1 Medical Center Dr., Lebanon, NH 03756.email: rjsinger@mac.com. 